\title{
Inteligencia emocional percibida y desgaste laboral en médicos internos de pregrado
}

\author{
R. Ortiz-Acosta, B.E. Beltrán-Jiménez
}

Objetivo. Analizar la relación entre los niveles de inteligencia emocional percibida y desgaste laboral en médicos internos de pregrado.

Sujetos y métodos. Para ello se utilizó la Trait Meta-Mood Scale, que evalúa la inteligencia emocional percibida, y el inventario de burnout de Maslach, en 44 médicos internos de pregrado de tres hospitales de los Servicios de Salud de Sonora.

Resultados. El factor de atención emocional se relaciona negativamente con agotamiento. Claridad correlaciona de manera positiva con reparación emocional y realización personal, y de forma negativa con agotamiento y despersonalización. Reparación de las emociones correlaciona de forma negativa con agotamiento y despersonalización, y de manera positiva con realización personal. Los análisis de regresión revelaron que el desgaste laboral del médico de pregrado se explicaba por una baja atención emocional y falta de claridad en sus emociones.

Conclusión. Estos hallazgos evidencian que ciertos factores emocionales deben tenerse en cuenta para explicar el desgaste laboral en el médico interno de pregrado.

Palabras clave. Desgaste laboral. Inteligencia emocional. Médico interno de pregrado.

\section{Perceived emotional intelligence and burnout in undergraduate medicine interns}

Aim. To analyze the relationship between the levels of perceived emotional intelligence and burnout in undergraduate medicine interns.

Subjects and methods. For this, we used the Trait Meta-Mood Scale, which evaluates perceived emotional intelligence, the Maslach Burnout Inventory, in 44 undergraduate interns from three hospitals of Sonora Health Services.

Results. The factor of emotional attention is related negatively with exhaustion. Clarity correlates in a positive way with emotional repair and self-fulfillment; and negatively with exhaustion and depersonalization. Emotional repair correlates in a negative way with exhaustion and depersonalization, and positively to self-fulfillment. Regression analyses showed that burnout in undergraduate interns was explained by a low emotional attention and a lack of clarity in their emotions.

Conclusion. Those findings prove that certain emotional factors should be taken into account to explain the burnout in undergraduate medicine interns.

Key words. Burnout. Emotional intelligence. Undergraduate medicine interns.

\section{Introducción}

El internado medico de pregrado generalmente se cursa en el quinto año de la carrera de Medicina, previo a la realización del servicio social, y una vez que el estudiante ha transitado por los cursos de las denominadas 'ciencias básicas' y por los cursos correspondientes a las 'ciencias clínicas', en este orden. Durante esta etapa, el médico interno transita por los servicios básicos de un hospital de segundo nivel. Se trata de un periodo con caracterís- ticas atípicas desde el punto de vista de las condiciones en que ocurre el proceso educativo, así como por el tipo de aprendizaje, eminentemente práctico que lo caracteriza, además de la permanencia a tiempo casi exclusivo del interno en el medio hospitalario [1]. Por esa razón, el internado representa la culminación de la enseñanza de pregrado de la medicina, un período de aprendizaje durante el cual se supone que el estudiante integra y aplica los conocimientos adquiridos en los cursos previos [2].
Servicios de Salud de Sonora. Hermosillo, Sonora, México.

Correspondencia:

Dr. Rogelio Ortiz Acosta. Departamento de Desarrollo Humano. Dirección General de Enseñanza y Calidad. Servicios de Salud de Sonora. Calzada de los Ángeles y Dr. José Miró Abella. Col. Las Quintas. CP 83240 Hermosillo, Sonora, México. E-mail: rogermeal854@hotmail.com Cㄷ 2011 Educación Médica 
Desde el punto de vista del programa de estudios, son estudiantes inscritos en una facultad de medicina, sujetos a su supervisión y evaluación. En la práctica, sin embargo, los internos ocupan una posición en la estructura de empleos del hospital. La importancia exacta de esa posición varía según las necesidades de cada hospital, el grado de control que ejerce la facultad de medicina, etc. De esa manera, en los hospitales donde no hay suficiente personal, los internos se ocupan de gran parte de las tareas de atención de los pacientes, mientras que en los hospitales con exceso de personal su función se reduce a la de personal auxiliar [2].

Un punto importante es el aprendizaje en el año de internado, para lo cual es necesario entender cómo se realiza el aprendizaje de las ciencias de la salud, el cual no puede ser conceptualizado sólo en términos cognitivos, sino que se deben agregar también los procedimientos y actitudes porque la educación médica debe perseguir un desarrollo completo y armónico de las personas, que incluya la promoción del pensamiento crítico y lo capacite para formarse opiniones propias o adoptar decisiones de manera independiente [3]. Los programas incluyen objetivos relacionados con contenidos actitudinales, como la formación científica, el desarrollo de valores éticos y de compromiso social, así como otros ubicados en esta misma línea que se relacionan con el desarrollo de habilidades para el trabajo en equipo y de relación con el paciente. Son objetivos que apuntan hacia una de las áreas esenciales de la formación médica: la socialización profesional. Sin embargo, este tipo de objetivos sólo aparecen enunciados sin que después se retomen en la metodología pedagógica ni como contenidos específicos. Esto es, con estos objetivos, se intuye dicha función de socialización profesional que cumple el internado, pero no se aborda de manera explícita ni se detalla cómo hacerlo [1].

Los profesionales de la salud, al trabajar con personas, ponen en juego más aspectos personales de los que generalmente se piensa, sobre todo a escala emocional, puesto que en muchas ocasiones no se puede evitar que el profesional quede afectado por las diferentes situaciones que presentan los pacientes. El fomento del desarrollo emocional del profesional sanitario no sólo debe favorecerse en el ámbito personal, también forma parte de grupos, de organizaciones y de la misma sociedad. En el sustrato cultural existe pánico para abordar el tema de las emociones; la mejor manera de empezar a tratarlo es desmitificándolo, informando y formando a los profesionales en ese aspecto [4].

\section{Inteligencia emocional}

Las emociones representan una fuente de información útil acerca de las relaciones que se establecen entre el individuo y su medio [5]. En la actualidad, el estudio de la emoción nos lleva al concepto de inteligencia emocional, entendida como la habilidad de percibir con exactitud, valorar y expresar emociones, la habilidad de acceder o generar sentimientos que faciliten el pensamiento, la habilidad de comprensión emocional y conocimiento emocional, y la habilidad de regular emociones para promover el crecimiento intelectual y emocional [6]. La inteligencia emocional plantea una nueva visión en el estudio de las emociones, las cuales han pasado de ser elementos perturbadores de los procesos cognitivos a considerarse fenómenos vitales del ser humano que proporcionan información útil para solucionar nuestros problemas cotidianos. Más aún, desde este acercamiento se considera que el uso inteligente de nuestras emociones puede ayudar a la regulación de nuestra adaptación psicológica y física [7].

\section{Desgaste laboral}

La relación entre el profesional de la salud y su cliente es una relación entre dos personas y, por tanto, está sujeta a los mismos condicionantes que toda relación humana. Trabajar con personas proporciona satisfacciones emocionales cuando se consiguen las metas terapéuticas marcadas, pero también puede producir insatisfacciones y generar emociones negativas [4]. Maslach fue quien primero empleó públicamente el término burnout para referirse a una situación que cada vez se hacía más frecuente entre aquellas personas que, por la naturaleza de su trabajo, debían mantener un contacto directo y continuado con la gente y después de meses o años de dedicación acababan por desgastarse profesionalmente $[8,9]$.

El burnout se desarrolla en aquellos profesionales cuyo objetivo de trabajo son personas y se compone de tres dimensiones:

- Agotamiento o cansancio emocional: es el cansancio o la fatiga que puede manifestarse física o psíquicamente, es la sensación descrita como no poder dar más de uno mismo a los demás.

- Despersonalización: consistente en el desarrollo de sentimientos, actitudes y respuestas negativas, distantes y frías hacia otras personas, especialmente hacia los beneficiarios del propio trabajo.

- Baja realización personal: tendencia a evaluar el propio trabajo de forma negativa, sentimientos de inadecuación y fracaso; surge cuando se verifica 
que las demandas que se le hacen exceden su capacidad para atenderlas de forma competente [10].

La aparición de estas emociones negativas suele llevar a una pérdida tanto de la calidad como del rendimiento del trabajo, que puede hacerse extensiva a la calidad de vida personal [4]. Por ello, nuestro estudio pretende explorar la relación entre inteligencia emocional percibida y desgaste laboral en médicos internos de pregrado, en tres hospitales de los Servicios de Salud de Sonora.

\section{Sujetos y métodos}

Se realizó un estudio transversal, descriptivo y de correlación en médicos internos de pregrado que habían ingresado en agosto del 2007, para concluirlo en julio del 2008. Los instrumentos de medición se aplicaron durante mayo y junio del 2008 a 44 médicos internos de pregrado adscritos a tres hospitales del sector público (Hospital General del Estado, Hospital General de Ciudad Obregón, Hospital General de Navojoa) pertenecientes a los Servicios de Salud de Sonora, México. La participación fue voluntaria, previo consentimiento informado, garantizando la confidencialidad y anonimato de los datos proporcionados. El análisis estadístico se realizó con el programa SSPS v. 10, empleando el coeficiente de correlación $r$ de Spearman y regresión jerárquica, con una significación de $p<0,01$ y $p<0,05$.

\section{Instrumentos de medición}

- Trait Meta-Mood Scale-24 (TMMS-24). La escala trata del metaconocimiento de los estados emocionales y evalúa, a través de 24 ítems, las diferencias individuales en las destrezas que las personas perciben, prestar atención a sus propias emociones, discriminar entre ellas y su capacidad percibida para regularlas. Se compone de 24 preguntas, ocho por dimensión, y su fiabilidad para cada componente es: atención, $\alpha=0,90$; claridad, $\alpha=$ 0,90 , y reparación, $\alpha=0,86$ (Tabla I) $[4,11]$.

- Inventario de burnout de Maslach (MBI). Los elementos del MBI están redactados de una manera directa para expresar unos sentimientos o actitudes personales. Consta de 22 ítems tipo Likert en forma de afirmaciones referidas a las actitudes, emociones y sentimientos que el profesional muestra hacia el trabajo. Evalúa tres subescalas: agotamiento emocional (9 ítems), despersonalización (5 ítems) y realización personal (8 ítems). En cuanto a las propiedades psicométricas del
Tabla I. Trait Meta-Mood Scale-24 (TMMS-24).

1. Presto mucha atención a mis sentimientos

2. Normalmente me preocupo mucho por lo que siento

3. Normalmente dedico tiempo a pensar en mis emociones

4. Pienso que merece la pena prestar atención a mis emociones y estado de ánimo

5. Dejo que mis sentimientos afecten a mis pensamientos

6. Pienso en mi estado de ánimo constantemente

7. A menudo pienso en mis sentimientos

8. Presto mucha atención a cómo me siento

9. Tengo claros mis sentimientos

10. Frecuentemente puedo definir mis sentimientos

11. Casi siempre sé cómo me siento

12. Normalmente conozco mis sentimientos sobre las personas

13. A menudo me doy cuenta de mis sentimientos en diferentes situaciones

14. Siempre puedo decir cómo me siento

15. A veces puedo decir cuáles son mis emociones

16. Puedo llegar a comprender mis sentimientos

17. Aunque a veces me siento triste suelo tener una visión optimista

18. Aunque me sienta mal procuro pensar en cosas agradables

19. Cuando estoy triste pienso en todos los placeres de la vida

20. Intento tener pensamientos positivos aunque me sienta mal

21. Si doy demasiadas vueltas a las cosas complicándolas trato de calmarme

22. Me preocupo por tener un buen estado de ánimo

23. Tengo mucha energía cuando me siento feliz

24. Cuando estoy enfadado intento cambiar mi estado de ánimo

Las respuestas se valoran de acuerdo con el siguiente baremo: 1 , nada de acuerdo; 2, algo de acuerdo; 3, bastante de acuerdo; 4, muy de acuerdo; 5 , totalmente de acuerdo.

MBI: agotamiento emocional, $\alpha=0,90$; despersonalización, $\alpha=0,79$, y realización personal, $\alpha=$ 0,071 (Tabla II) [12]. 
Tabla II. Inventario de burnout de Maslach.

1. Me siento emocionalmente agotado por mi trabajo

2. Me siento cansado al final de la jornada de trabajo

3. Me siento fatigado cuando me levanto por la mañana y tengo que ir a trabajar

4. Comprendo fácilmente cómo se sienten los pacientes

5. Trato a algunos pacientes como si fueran objetos impersonales

6. Trabajar todo el día con mucha gente es un esfuerzo

7. Trato muy eficazmente los problemas de los pacientes

8. Me siento agotado por mi trabajo

9. Creo que influyo positivamente con mi trabajo en la vida de las personas

10. Me he vuelto más insensible con la gente desde que ejerzo esta profesión

11. Me preocupa el hecho de que este trabajo me endurezca emocionalmente

12. Me siento muy activo en mi trabajo

13. Me siento frustrado en mi trabajo

14. Creo que estoy trabajando demasiado

15. Realmente no me preocupa lo que le ocurre a mis pacientes

16. Trabajar directamente con personas me produce estrés

17. Puedo crear fácilmente una atmósfera relajada con mis pacientes

18. Me siento estimulado después de trabajar con mis pacientes

19. He conseguido muchas cosas útiles en mi profesión

20. Me siento acabado

21. En mi trabajo trato los problemas emocionales con mucha calma

22. Siento que los pacientes me culpan de alguno de sus problemas

Las respuestas se valoran de acuerdo con el siguiente baremo: 0 , nunca; 1 , pocas veces al año o menos; 2 , una vez al mes o menos; 3 , unas pocas veces al mes o menos; 4 , una vez a la semana; 5 , pocas veces a la semana; 6 , diariamente.

\section{Resultados}

La muestra final se compone de 44 médicos internos de pregrado, con una media de edad de 23 años (rango: $21-26$ años) y un $57 \%$ de mujeres. En rela-
Tabla III. Características sociodemográficas.

\begin{tabular}{|c|c|c|c|}
\hline & & $n$ & $\%$ \\
\hline \multirow{2}{*}{ Sexo } & Femenino & 25 & 57 \\
\hline & Masculino & 19 & 43 \\
\hline \multirow{2}{*}{$\begin{array}{l}\text { Estado } \\
\text { civil }\end{array}$} & Solteros & 41 & 93 \\
\hline & Casados & 3 & 7 \\
\hline \multirow{3}{*}{$\begin{array}{l}\text { Hospital } \\
\text { sede }\end{array}$} & Hospital General de Estado & 30 & 68 \\
\hline & Hospital General de Navojoa & 4 & 9 \\
\hline & $\begin{array}{l}\text { Hospital General } \\
\text { de Ciudad Obregón }\end{array}$ & 10 & 23 \\
\hline
\end{tabular}

ción al estado civil, el 93\% era soltero, y el 7\%, casado. El $68 \%$ de la población estudiada pertenece al Hospital General del Estado de Sonora, el 23\% al Hospital General de Ciudad Obregón, y el 9\% al Hospital General de Navojoa (Tabla III).

\section{Resultados de inteligencia emocional percibida y desgaste laboral}

Se observa en la tabla IV que atención emocional correlaciona positivamente con claridad emocional $(r=0,37 ; p<0,01)$ y de manera negativa con agotamiento emocional $(r=-0,47 ; p<0,001)$. Claridad emocional correlacionó de manera positiva con reparación de las emociones $(r=0,57 ; p<$ $0,001)$ y realización personal $(r=0,72 ; p<0,001)$, y se encontraron correlaciones negativas con agotamiento emocional $(r=-0,63 ; p<0,001)$ y despersonalización $(r=-0,49 ; p<0,001)$. Reparación de las emociones correlacionó de forma negativa con agotamiento emocional $(r=-0,49 ; p<0,001)$ y despersonalización $(r=-0,28 ; p<0,001)$, y de manera positiva con realización personal $(r=0,57 ; p<$ $0,001)$. Agotamiento emocional correlacionó positivamente con despersonalización $(r=0,55 ; p<$ $0,001)$ y negativamente con realización personal $(r=-0,60 ; p<0,001)$. Despersonalización correlacionó de forma negativa con realización personal $(r=-0,40 ; p<0,001)$.

\section{Resultados del análisis de regresión} de inteligencia emocional y desgaste laboral

Para comprobar la relación existente entre los com- 
ponentes de la inteligencia emocional percibida y las dimensiones del burnout, se realizaron diferentes análisis de regresión jerárquicos, analizándose las relaciones de los factores del burnout con las dimensiones de la inteligencia emocional percibida. Las variables dependientes fueron agotamiento emocional, despersonalización y realización personal. En la tabla V es posible apreciar cómo estos análisis confirman los resultados de las correlaciones antes presentadas. Respecto al agotamiento emocional como variable dependiente, se obtuvieron resultados significativos $(F=10,12 ; p<0,000)$, explicando la dimensión de atención emocional un $57 \%$ de la varianza $(B=-0,23 ; p<0,05)$. Cuando se introduce como variable dependiente la despersonalización $(F=4,24 ; p<0,01)$, un $24 \%$ de la varianza se explicó por la claridad emocional como predictor negativo $(B=0,47 ; p<0,00)$. Por último, para el factor de realización personal $(F=17,34 ; p<0,00)$ como variable dependiente, un $56 \%$ de la varianza se explicó por la claridad emocional $(B=0,57 ; p<0,00)$.

\section{Discusión}

Dentro de la literatura científica existen pocos estudios que revelen el estado emocional del médico interno de pregrado; el presente trabajo pretendía mostrar la relación existente entre la inteligencia emocional percibida y el desgaste laboral en médicos que estaban por concluir su internado de pregrado.

Los análisis correlacionales de inteligencia emocional percibida en el componente de atención emocional revelan que, cuando el médico interno de pregrado posee niveles adecuados en atención emocional, con tendencia a observar y pensar sobre sus emociones y sentimientos, se incrementa la claridad de estas emociones. Estos resultados constatan lo publicado por Thayer et al [13], quienes encontraron que niveles medios de atención acompañados de una alta claridad se relacionarían con un proceso de la información emocional más adecuado y con mejores niveles de ajuste.

La relación negativa entre atención de las emociones y agotamiento emocional revela que un nivel excesivamente bajo de atención a las emociones no permitirá el acceso a la información que éstas proporcionan, perjudicando así su comprensión y regulación [14], por lo que sobrevendrá en el médico de pregrado un mayor agotamiento emocional. Respecto a la dimensión de claridad emocional, se relaciona con reparación emocional, lo cual indica que los médicos internos de pregrado que fácilmente identifican una emoción específica durante situa-
Tabla IV. Estadísticos descriptivos y correlación, inteligencia emocional y desgaste laboral.

\begin{tabular}{|c|c|c|c|c|c|c|}
\hline & 1 & 2 & 3 & 4 & 5 & 6 \\
\hline \multicolumn{7}{|l|}{ 1. Atención } \\
\hline 2. Claridad & $0,37^{\mathrm{a}}$ & & & & & \\
\hline 3. Reparación & $-0,20$ & $0,57^{b}$ & & & & \\
\hline 4. Agotamiento laboral & $-0,47^{b}$ & $-0,63^{b}$ & $-0,49^{b}$ & & & \\
\hline 5. Despersonalización & $-0,21$ & $-0,49 b$ & $-0,28^{b}$ & $0,55^{b}$ & & \\
\hline 6. Realización personal & 0,33 & $0,72^{b}$ & $0,57^{b}$ & $-0,60^{b}$ & $-0,40^{b}$ & \\
\hline
\end{tabular}

Tabla V. Análisis de regresión sobre las dimensiones de desgaste laboral e inteligencia emocional percibida.

\begin{tabular}{|c|c|c|c|c|c|}
\hline & $\% R^{2}$ & $F$ & $p$ & $B$ & $p$ \\
\hline Agotamiento emocional & $57 \%$ & 10,1 & 0,00 & & \\
\hline Atención & & & & $-0,23$ & $0,05^{a}$ \\
\hline Claridad & & & & $-0,20$ & 0,24 \\
\hline Reparación & & & & $-0,09$ & 0,50 \\
\hline Despersonalización & $24 \%$ & 4,24 & 0,01 & & \\
\hline Atención & & & & $-0,04$ & 0,78 \\
\hline Claridad & & & & $-0,47$ & $0,00^{b}$ \\
\hline Reparación & & & & 0,00 & 0,99 \\
\hline Realización personal & $56 \%$ & 17,3 & 0,00 & & \\
\hline Atención & & & & 0,04 & 0,72 \\
\hline Claridad & & & & 0,57 & $0,00^{b}$ \\
\hline Reparación & & & & 0,23 & 0,07 \\
\hline
\end{tabular}

${ }^{\mathrm{a}} p<0,05 ;{ }^{\mathrm{b}} p<0,001$.

ciones de estrés pasarán menos tiempo atendiendo a sus reacciones emocionales, invirtiendo además menos recursos cognitivos, lo cual les permitirá evaluar las alternativas de acción, mantener sus pensamientos en otras tareas o bien llevar a cabo estrategias de afrontamiento más adaptativas [15]. En este sentido, la claridad se ha relacionado positivamente con realización personal, es decir, el médico interno de pregrado que es capaz de percibir claramente sus emociones, que sabe lo que está sintiendo, presentará una mayor realización personal, lo cual concuerda con lo comunicado en otra investigación [16] que indica que los profesionales de la 
salud con una buena claridad en sus emociones denotan un mayor nivel de realización personal. Las personas con una alta claridad emocional no sólo tienen la creencia de saber si su estado de ánimo es positivo o negativo, sino que se perciben como más capaces de comprender qué emociones están experimentando, cómo se manifiestan tales emociones o cuáles son sus causas y consecuencias [14]. En cambio, el médico de pregrado que se encuentra confundido en sus emociones se sentirá más agotado emocionalmente y presentará mayores índices de despersonalización y actitudes cínicas, distantes y frías hacia los usuarios de los servicios, con irritabilidad y pérdida de motivación hacia su trabajo. Ello corrobora lo encontrado en otro estudio, que señala que una persona con baja claridad emocional, que está confundida sobre sus emociones, es más probable que realice reacciones impredecibles y a menudo problemáticas ante situaciones emocionales [15]; de igual forma [17], plantean que aquellas personas que tienen sus emociones poco claras presentan más sentimientos y actitudes negativas y cínicas hacia los usuarios.

En el componente de reparación emocional se encontraron correlaciones negativas con agotamiento emocional, es decir, cuando al médico interno de pregrado se le dificulta interrumpir sus estados emocionales negativos y prolongar los positivos, se incrementa el agotamiento emocional, lo que concuerda con otros resultados [18] indicativos de que las personas que presentan mayor cansancio emocional, manifiestan disminución en el autoconocimiento y expresión de las emociones, y muestran menor capacidad de reparación emocional. Por otro lado, se observa que reparación de las emociones correlacionó positivamente con realización personal, lo que revela que los médicos de pregrado que son capaces de pasar de un estado emocional negativo a uno positivo presentan mayor realización personal. Estos resultados son congruentes con otros autores [16,19], quienes indican que una capacidad más elevada para reparar y discriminar sus estados emocionales denota un mayor nivel de realización personal, lo que aumenta la satisfacción vital de las personas tras controlar sus niveles anímicos. Así mismo, Cleries et al [4] muestran que, ser capaz, por parte del profesional, de prestar atención empática a los demás no es una habilidad gratuita o una gracia especial; es, por el contrario, una habilidad que se relaciona con la capacidad de comunicación y la habilidad de percepción de uno mismo. Sentirse a gusto consigo mismo es la condición necesaria para relacionarse de forma efectiva con los demás.
En conjunto, los análisis de correlación y de regresión confirman los resultados obtenidos y añaden evidencia acerca de la capacidad predictiva de la inteligencia emocional percibida en la explicación del desgaste laboral. La dimensión de atención emocional se ha mostrado como predictor significativo de forma negativa del agotamiento emocional; así, el médico de pregrado con baja capacidad de atención a sus emociones presenta mayores índices de agotamiento emocional, lo que revela que la persona que muestra escasa atención a sus emociones, aquella que no considera relevante sus estados afectivos, no utilizará probablemente esa información para regular su estado anímico, lo cual conllevará un agotamiento emocional

Así mismo, el factor de despersonalización fue explicado negativamente por la claridad emocional. En este sentido, a la hora de explicar el proceso de despersonalización en el médico de pregrado, éste queda mediado por la dificultad para distinguir y describir las emociones que cotidianamente experimenta la persona. Por último, para la dimensión de realización personal se observa como predictor positivo la claridad emocional, lo cual revela que, para el profesional médico de pregrado, contar con una adecuada claridad en sus emociones, que se percibe como más capaz de comprender qué emociones está sintiendo, indica un mayor nivel de realización personal.

En conclusión, los hallazgos de nuestro estudio ponen de manifiesto la importancia de la inteligencia emocional percibida en el médico interno de pregrado porque permite observar una estrecha relación entre la aparición del desgaste laboral o burnout y una baja atención a sus emociones, poca comprensión de sus estados emocionales y dificultades para interrumpir emociones negativas y prolongar las positivas.

Lo anterior tendría que considerarse en la formación del profesional médico. La adquisición de conocimientos y de procedimientos clínicos son herramientas indispensables para su futuro desempeño. Es necesario, también, integrar el desarrollo de habilidades emocionales y el autoconocimiento del profesional en formación, todo desde un enfoque sistémico, que logre conjuntar una instrucción académica desde el saber, el hacer y el sentir. Propiciar el desarrollo de la capacidad del médico interno de pregrado para identificar, comprender y regular sus estados emocionales es indispensable porque dichas habilidades le permitirán vislumbrar desde una perspectiva más integral su labor asistencial, preparándolo para un mejor afrontamiento de las complejidades del quehacer médico. 
Bibliografía

1. Flores E, Gasca GG, Urbina GR, Ríos CV, Lloret RA. Nuevos modelos educativos en el internado médico de pregrado. La participación de la universidad. Reencuentro 2005; 42: 1-17.

2. Frenk J. La atención médica, la enseñanza de la medicina y el mercado de trabajo para los médicos: el internado de pregrado. Rev Med Salud 1984; 18: 329-43.

3. Tapia VR, Núñez TR, Salas PR, Rodríguez-Orozco A. El internado médico de pregrado y las competencias clínicas. México en el contexto latinoamericano. Educ Med Super 2007; 21: 4 .

4. Cleries X, Raya MA, Kronfly E, Escoda JJ, Cases C. Hacia el aprendizaje de las emociones y sistemas motivacionales en la relación asistencial. Educ Med 2002; 5: 113-25.

5. Salovey P, Mayer ID, Goldman SL, Turvey C, Palafai TP. Emotional attention, clarity, and repair: exploring emotional intelligence using the Trait Meta-Mood Scale. In Pannebaker JW, ed. Emotion, disclosure, and health. Washington DC: Psychological Association; 1995. p. 159-71.

6. Mayer JD, Salovey P. What is emotional intelligence? In Salovey P, Sluyter D, eds. Emotional development and emotional intelligence: implications for educators. New York: Basic Books; 1997. p. 3-31.

7. Extremera N, Fernández B. El papel de la inteligencia emocional en el alumnado: evidencias empíricas. Revista Electrónica de Investigación Educativa 2004; 6: 2 .

8. Maslach C. Burnout: the cost of caring. Englewood Cliffs, NY: Prentice Hall; 1982.

9. Spear LW, Sudarshan CH, Devis A, Nelson J. Job satisfaction and burnout in mental health services for older people. Australas Psychiatry 2004; 12: 58-65.
10. Guerrero BE. Análisis pormenorizado de los grados de burnout y técnicas de afrontamiento del estrés docente en el profesorado universitario. Anales de Psicología 2003; 19: 145-58.

11. Fernández-Berrocal P, Extremera N, Ramos N. Validity and reliability of the Spanish modified version of the Trait MetaMood Scale. Psychol Rep 2004; 94: 751-5.

12. Maslach C, Jackson SE. MBI-Inventario Burnout de Maslach. Madrid: TEA; 1997.

13. Thayer JF, Rossy LA, Ruiz-Padial E, Johnsen BH. Gender in the relationship between emotional regulation and depressive symptoms. Cognit Ther Res 2003; 27: 349-64.

14. Salguero JM, Iruarrizaga DI. Relaciones entre inteligencia emocional percibida y emocionalidad negativa: ansiedad, ira, tristeza/depresión. Ansiedad y Estrés 2006; 12: 207-221.

15. Gohm CL, Clore GL. Four latent traits of emotional experience an their involvement in attributional style, coping and well-being. Cognition \& Emotion 2002; 16: 495-518

16. Augusto-Landa JM, Berrios-Martos MP, López-Zafra E, Aguilar-Luzón MP. Relación entre burnout e inteligencia emocional y su impacto en salud mental, bienestar y satisfacción laboral en profesionales de enfermería. Ansiedad y Estrés 2006; 12: 479-93.

17. Nogueira R, Gabari JM. Relación entre inteligencia emocional, asertividad y burnout. Congreso Universitario de Psicología y Logopedia. 30 de noviembre-2 de diciembre de 2005.

18. Extremera N, Férnandez-Berrocal P, Durán A. Inteligencia emocional y burnout en profesores. Encuentros de Psicología Social 2003; 1: 260-5.

19. Extremera N, Fernández B. Examen de las relaciones entre inteligencia emocional, salud mental y burnout en profesores de secundaria, un estudio piloto. Interpsiquis 2005 . 